Trauma Berufskrankh 2017· [Suppl 1]: 19:S101-S108 G. Fitze

DOI 10.1007/s10039-017-0231-4

Online publiziert: 9. Februar 2017

Klinik für Kinderchirurgie, Universitätsklinikum Dresden, Dresden, Deutschland

๑) Springer Medizin Verlag Berlin 2017

CrossMark

\title{
Abdominal- und Thoraxtrauma des Kindes
}

\section{Abdominaltrauma}

\section{Epidemiologie}

Entsprechend den Ergebnissen der amtlichen Statistik zum Verletzungsgeschehen 2012, publiziert durch das Statistische Bundesamt im Jahr 2014, stellen die Folgen eines Unfalles im Alter jenseits des 1. Geburtstages bis zum 20. Lebensjahr die häufigste Ursache für das Versterben eines jungen Menschen dar. Konkret bedeutet dies, dass im Jahr 2012 in der Bundesrepublik 890 Kinderunfälle mit einem letalen Ausgang registriert worden sind. Dabei waren 308 Kinder im Alter bis zum 15. Lebensjahr betroffen und $582 \mathrm{im}$ Alter zwischen 15 und 19 Jahren. Unabhängig von der Schwere der Unfallfolgen werden in Deutschland aktuell täglich 770 Kinder wegen der Folgen eines Unfalls stationär aufgenommen und behandelt. Dabei werden Abdominalverletzungen mit einer Häufigkeit zwischen 2 und $5 \%$ registriert. Typischerweise handelt es sich dabei in Europa zu über $90 \%$ um stumpfe Gewalteinwirkungen auf den Bauchraum. Die penetrierenden Verletzungen der Abdominalhöhle stellen eine Rarität dar. Einer Publikation von Wessel et al. [24] aus dem Jahr 2000 zufolge sind die Nieren das am häufigsten verletzte Organ, gefolgt von der Milz und der Leber sowie der Verletzung des Magen-Darm-Traktes. Am seltensten sind Pankreasverletzungen beobachtet worden. Diese Angaben basieren auf der Auswertung von Patienten mit einem stumpfen Bauchtrauma über einen

Dieser Beitrag ist eine überarbeitete und erweiterte Version des Beitrages: Fitze $G$ (2016) Management der Organverletzungen bei Adoleszenten. Trauma und Berufskrankheit, Suppl.2.DOI 10.1007/s10039-015-0056-y.
Zeitraum von 20 Jahren. Dabei stellte sich heraus, dass knapp die Hälfte dieser Patienten ihr stumpfes Bauchtrauma im Rahmen eines Verkehrsunfalles erlitten hat. Allgemein ist jedoch davon auszugehen, dass beim stumpfen Bauchtrauma im Kindesalter in ca. $20-25 \%$ eine Organverletzung im Abdominalraum zu diagnostizieren ist.

\section{Bildgebende Diagnostik}

Neben der Anamnese, dem klinischen Untersuchungsbefund und Erhebung spezifisch pathologischer Laborparameter (Erhöhung der Transaminasen bei Leberverletzungen, Erhöhung der Lipase und Amylase bei Pankreasverletzungen, einer Hämaturie bei Nierenverletzungen und einer möglichen Leukozytose bei Milzverletzungen) kommt der bildgebenden Diagnostik im Rahmen der Abklärung eines stumpfen Bauchtraumas eine essenzielle Bedeutung zu. In Europa wird dabei als Primärdiagnostik die Sonographie des Abdomens klar präferiert. Hinsichtlich des Nachweises von freier Flüssigkeit im Abdominalraum ist durch diese Untersuchung eine Sensitivität von über $90 \%$ bei einer Spezifität von bis zu $97 \%$ gegeben. Große Mengen freier Flüssigkeit korrelieren eher mit einer Milz- als mit einer Leberverletzung. Umgekehrt schließt das Fehlen von freier Abdominalraumflüssigkeit jedoch eine Verletzung parenchymatöser Bauchorgane nicht aus. Als wesentlicher Vorteil dieser Untersuchungsmethode ist die kurzfristige Wiederholbarkeit und somit das Monitoring der Befunde am Krankenbett zu werten.

Im Gegensatz zu dieser Strategie wird insbesondere in den USA als Primärdiagnostik bei Vorliegen eines stumpfen
Bauchtraumas die Kontrastmittel-Computertomographie (CT) angewendet. Bei einer Sensitivität und Spezifität hinsichtlich des Nachweises einer Organverletzung von knapp $100 \%$ ist sie als die sicherste Methode und als Goldstandard anzusehen. Auf der CT-Diagnostik basiert außerdem die Schweregradeinteilung der parenchymatösen Organverletzungen des Bauchraumes. Sie zeichnet sich durch einen äußerst geringen Zeitaufwand bei kaum vorhandener Abhängigkeit der Befunde vom jeweiligen Untersucher aus. Somit werden Verletzungen in weniger als $2,5 \%$ der Fälle übersehen. Im Rahmen der aktuellen Diskussion bezüglich der Strahlenexposition im Kindesalter durch eine CT-Untersuchung ist dabei unbedingt eine kindgerechte Adaptation der Untersuchungsmodalitäten $\mathrm{zu}$ fordern. In einer gerade publizierten niederländischen Studie konnte gezeigt werden, dass bei 64 hämodynamisch stabilen Kindern nach Abdominaltrauma die CT-Untersuchung nur bei 3 Patienten zu einer Änderung der Diagnosen und des therapeutischen Managements geführt hat [18]. So kommen die Autoren zu dem Fazit, dass die Indikation zur Durchführung einer Kontrastmittel-CT im Kindesalter auf einer klar formulierten, auf dem klinischen Untersuchungsbefund basierenden Fragestellung beruhen sollte, die eine therapeutische Konsequenz impliziert. Unter diesem Aspekt wird dem Grundsatz der rechtfertigenden Indikation zur Anwendung von Röntgenstrahlung auch auf diesem Gebiet Rechnung getragen und ist in Analogie zu den Prinzipien zur Durchführung der Polytraumaspirale im Kindesalter zu sehen.

Neben dem hohen Stellenwert der Radiologie im Rahmen der Diagnostik eines 

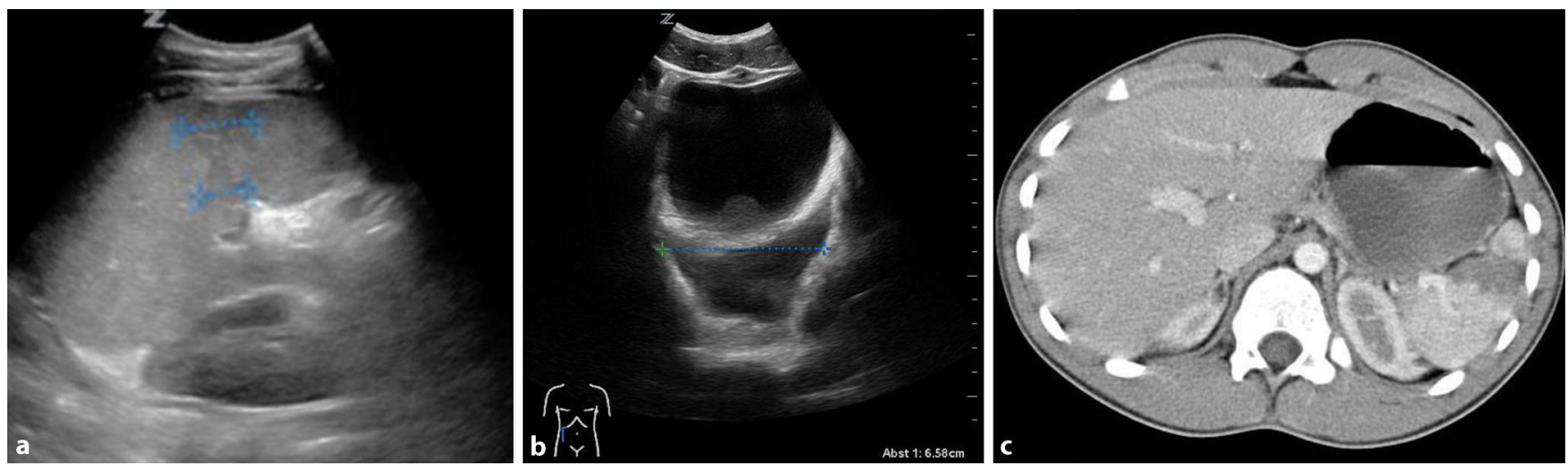

Abb. 1 ॥ 13-jähriger Junge, Zustand nach Sturz vom Baum aus ca. 3 m Höhe (aus [5]). a Sonographischer Nachweis einer zentralen Milzruptur mit wenig perisplenischer freier Flüssigkeit. b Typisch für eine Milzruptur ist die sonographisch nachgewiesene reichliche freie Flüssigkeit im Abdomen, hier retrovesikal im Rahmen der Erstuntersuchung.cDieComputertomographie bei Sturz aus großer Höhe zeigt neben der Milzruptur Grad IV eine Nierenkontusion links und eine Fraktur der 9. Rippe links

stumpfen Bauchtraumas im Kindesalter hat sich in den letzten Jahren auch die interventionelle Radiologie als therapeutische Option im Kindesalter etabliert. In ersten Studien hat sie sich als sichere und effektive Methode bei der Behandlung von persistierenden Blutungen der Milz, der Leber und der Niere erwiesen [11]. Limitierte eigene Erfahrungen (2 Patienten) konnten diese Berichte bei der Versorgung von Nierenrupturen bestätigen.

\section{Milz- und Leberverletzungen}

Im Kindesalter gibt es für die Verletzung der parenchymatösen Oberbauchorgane Milz und Leber bei einem stumpfen Bauchtrauma verschiedene prädisponierende Faktoren. Einerseits sind beide Organe durch ihre anatomischen Gegebenheiten in den Zwerchfellkuppeln sehr gut fixiert. Durch den relativ hohen Flüssigkeitsanteil und den höheren Anteil an elastischen Fasern im Grundgerüst der Gewebe besitzen sie eine hohe Organspannung, andererseits ist der Thorax im Kindesalter noch in hohem Maße verformbar. Somit kann bei einem stumpfen Bauchtrauma direkt die kinetische Energie auf beide Organe übertragen werden, die somit im Kindesalter leichter verletzt werden können. Bei ausgedehnten Leberverletzungen ist durch die Mitbeteiligung der großen venösen Gefäße im Kindesalter eine Mortalität von teilweise über $50 \%$ beschrieben. Das für die Milz beschriebene Phänomen der zweizeitigen Milz- ruptur wird zwar auch im Kindesalter kasuistisch beschrieben, wird aber insgesamt äußerst selten beobachtet.

Bereits vor 75 Jahren wurde am Hospital for Sick Children in Toronto das Prinzip des Non-Operative-Managements von parenchymatösen Organverletzungen im Kindesalter entwickelt. Diese Behandlung basiert auf der Möglichkeit des Sistierens von Blutungen durch Selbstkompression im begrenzten abdominalen Raum. Voraussetzungen sind dafür ein suffizientes Monitoring der Vitalparameter sowie die Möglichkeit zur engmaschigen Kontrolle von Laborparametern und $\mathrm{zu}$ dem bereits erwähnten Monitoring sonographischer Befunde am Krankenbett. Kreislaufstabilisierende Maßnahmen durch die Anwendung von Katecholaminen sowie Substitution von Flüssigkeitsverlusten bis hin zur Transfusion sind dabei fester Bestandteil der Behandlung. Bei der Transfusion von Erythrozytenkonzentraten wird dabei eine obere Grenze von $25 \mathrm{ml} / \mathrm{kg}$ Körpergewicht in den ersten $2 \mathrm{~h} \mathrm{bzw}$. von $40 \mathrm{ml} / \mathrm{kg}$ in $24 \mathrm{~h}$ toleriert. Die immer wieder postulierte Vermutung, dass im Gegensatz dazu durch ein operatives Vorgehen auf Transfusionen verzichtet werden könnte bzw. die transfundierten Mengen deutlich niedriger liegen als beim nichtoperativen Vorgehen, konnte durch Studien nicht bestätigt werden. So zeigten die Analysen von 205 Kindern mit einem Durchschnittsalter von 7 Jahren, von denen $17 \%$ operativ aufgrund einer abdomina- len Organverletzung behandelt wurden, einen signifikant höheren Transfusionsbedarf, eine längere Hospitalisierung und eine höhere Komplikationsrate [19].

In diesem Gesamtkontext hat sich in den letzten Jahrzehnten das Konzept des nichtoperativen Managements der Milzund Leberverletzungen im Kindesalter zunehmend durchgesetzt (• Abb. 1, 2 und 3). Die Effektivität und die Überlegenheit dieses Konzeptes konnten in vielen Studien signifikant belegt werden. Exemplarisch sei diesbezüglich die Studie von Frumiento und Vane [7] aus dem Jahr 2000 genannt. Von 267 Patienten mit einer isolierten Milzverletzung wurden in den Jahren zwischen 1985 und 1989 nur $21 \%$ nicht operativ versorgt. Diese Zahl stieg in den Jahren zwischen 1990 und 1995 auf $64 \%$ an, wobei ein überproportionaler Anteil in pädiatrisch spezialisierten Traumazentren konservativ behandelt werden konnte. Die Langzeitanalyse der Daten von Kindern mit isolierter Milzverletzung aus der Klinik, die das Prinzip des nichtoperativen Managements inauguriert hat, zeigt in den Jahren von 1956 bis 2006 einen Anstieg der nicht operierten Kinder von 42 auf $97 \%$ [3]. Bei $99 \%$ der Patienten konnte dabei eine Milzerhaltung erzielt werden. Die Transfusionen sanken von 60 auf $1 \%$ und die Mortalität bei isolierter Milzverletzung von 8 auf 0,7\%. Analog wurde das nichtoperative Management von Milzverletzungen auf Verletzungen der Leber übertragen. In einer Analyse von Nellensteijn et al. [17] aus dem Jahr 
2009 konnten diese zeigen, dass vor dem Jahr $200063 \%$ aller Patienten jünger als 18 Jahre mit einer Leberverletzung konservativ behandelt werden konnten, während in der Population nach 2000 in $84 \%$ keine Operationen stattfanden. Von diesen Patienten konnten $96 \%$ ohne Operation erfolgreich zu Ende behandelt werden. In einer Studie von Stylianos [23] aus dem Jahr 2000 wurde der Behandlungsverlauf von 312 Kindern aus 2 Jahren mit isolierter Leber- oder Milzverletzung der Stadien 1 bis 4 analysiert. Dabei sind innerhalb der ersten $48 \mathrm{~h}$ nach dem Unfall nur 4 Kinder (1,3\%) operativ versorgt worden. Von Stylianos wird dabei ein sehr kurzer stationärer Beobachtungszeitraum propagiert, der bei Stadium-IV-Verletzungen nur 5 Tage beträgt. Bei diesem Behandlungsregime kam es nur bei 6 Kindern $(1,0 \%) \mathrm{zu}$ einer Wiederaufnahme ins Krankenhaus, wobei auch in diesen Fällen keine Operation notwendig wurde.

Sollte jedoch die Indikation für eine operative Versorgung gestellt werden, so kommt dem Organerhalt höchste Priorität zu. Auch im Kindesalter ist zur Kontrolle von Blutungen das Packing der Resektion vorzuziehen. Vielfältige operative Techniken finden unter dem Aspekt des Organerhalts heute Anwendung. Genannt seien die Organkompression durch die Verwendung von vorgefertigten Netzen, einfache Kapselnähte, aber auch die Applikation von Hämostyptika oder die Oberflächenkoagulation mittels Infrarot- oder Laseranwendung. Durch selektive Gefäßligaturen lässt sich eine Kontrolle der Blutung mit anschließender Organteilresektion erzielen. Die Splenektomie ist prinzipiell als Ultima Ratio anzusehen. Kleinere operative Eingriffe sowie laparoskopische Interventionen sind bei Versorgung von Milzoder Leberverletzungen im Kindesalter entbehrlich und somit nicht indiziert.

\section{Nierenverletzungen}

Wie bereits einleitend beschrieben, ist die Niere das am häufigsten verletzte Organ beim stumpfen Bauchtrauma im Kindesalter. Insbesondere durch den anatomisch begrenzten Raum des Retroperitoneums ist für Verletzungen der Nie-

Trauma Berufskrankh 2017 • [Suppl 1]: 19:S101-S108 DOI 10.1007/s10039-017-0231-4

(c) Springer Medizin Verlag Berlin 2017

\section{G. Fitze}

\section{Abdominal- und Thoraxtrauma des Kindes}

\section{Zusammenfassung}

Unfälle sind im Alter zwischen dem 1. und 20. Lebensjahr die häufigste Todesursache. Das Abdomen ist in bis zu $5 \%$ der Fälle betroffen, und in bis zu $20 \%$ ist bei dem stumpfen Bauchtrauma eine Organverletzung zu diagnostizieren. Das Thoraxtrauma stellt im Kindesalter eine relativ seltene Verletzung dar, wobei die Lungenkontusion die häufigste Verletzung der Lunge im Kindesalter ist. Im Rahmen der bildgebenden Diagnostik hat im Kindesalter die Sonographie die größte Bedeutung. Die Indikation zum Kontrastmittel-CT des Abdomens muss kritisch gestellt werden. Mit wenigen Ausnahmen hat sich für diese Verletzungen im Kindesalter ein nichtoperatives Management durchgesetzt. Dieses Vorgehen ist für die isolierten Verletzungen der Milz und Leber evaluiert und setzt sich zunehmend ebenfalls für Verletzungen der Niere durch. Für Verletzungen des Pankreas ist bei nicht verletztem Pankreasgang eine konservative Therapie ebenfalls erfolgreich. Dagegen stellen Verletzungen des Gastrointestinaltraktes fast immer eine Indikation zur Laparotomie dar. Allgemein sollte die Laparotomie aber vermieden werden. Der Organerhalt hat das absolute Primat. Indikationen für eine primäre Operation stellen Kreislaufinstabilität, nachgewiesene Perforation eines Hohlorganes oder Peritonitis dar. Während die Mehrzahl der Thoraxverletzungen wie Rippen- oder Sternumfrakturen sowie Lungenkontusionen leichteren Charakter haben und in der Regel keiner operativen Intervention bedürfen, kommen selten schwere Verletzungen des Tracheobronchialsystems, penetrierende Thoraxverletzungen oder Hämatopneumothoraxe vor. Diese Verletzungen sind meist als Notfälle operativ zu versorgen. Trotzdem sind diese Traumata wegen der Kombination mit meist schweren Begleitverletzungen mit einer hohen Letalität assoziiert.

\section{Schlüsselwörter}

Stumpfes Bauchtrauma - Thoraxtrauma . Diagnostik · Organverletzungen · Therapie

\section{Pediatric abdominal and thoracic trauma}

\section{Abstract}

Accidents are the main cause of death between the ages of 1 and 20 years. The abdomen is affected in up to $5 \%$ of cases and blunt abdominal trauma resulting in organ injuries is diagnosed in up to $20 \%$. Thoracic trauma is a relatively uncommon injury in childhood, whereby lung contusions are the most frequent injuries of the lungs in childhood. In imaging diagnostics ultrasonography plays the most important role in childhood. The indications for abdominal contrast-enhanced computed tomography (CT) should be critically evaluated. With few exceptions, nonoperative management is the method of choice for these injuries in childhood. This approach has been evaluated for isolated injuries of the liver and spleen and is being used increasingly more for injuries of the kidneys. For injuries to the pancreas nonoperative management is also successful if the pancreatic duct is not involved. In contrast, injuries of the gastrointestinal tract nearly always necessitate a primary laparotomy; however, in general a laparotomy should be avoided. Preservation of organs is the primary aim of therapy. The indications for a primary surgical approach are circulation instability, perforation of an abdominal organ and peritonitis. Although the majority of thoracic injuries, such as fractures of the ribs and sternum as well as lung contusions are of moderate character and as a rule do not require surgical therapy, less common but more severe injuries in the tracheobronchial system, penetrating chest wounds and hematopneumothorax generally require emergency surgical interventions and are often associated with higher mortality due to mostly severe concomitant injuries.

\section{Keywords}

Blunt abdominal trauma - Thoracic trauma . Diagnostics · Organic injuries · Treatment 

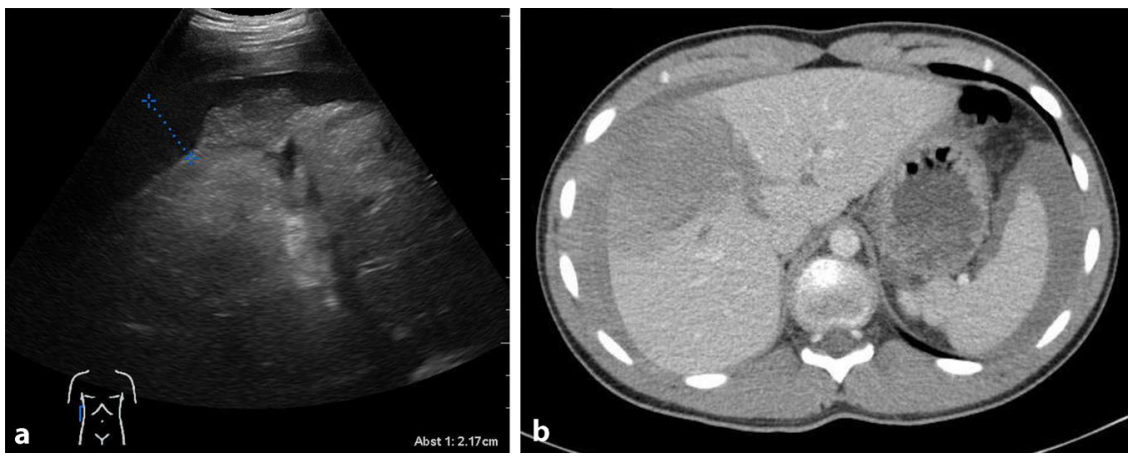

Abb. 2 \ De 15-jährige Junge ist mit dem BMX-Rad aus dem Bus gefahren, gestürzt und hat sich den Lenker in den rechten Oberbauch gerammt (aus [5]). a Sonographisch zeigt sich eine Leberruptur am Übergang vom rechten zum linken Leberlappen mit einer ausgedehnten Kontusionszone und reichlich freier Flüssigkeit im rechten Oberbauch. b Die Kontrastmittelcomputertomographie des Abdomens zeigt neben der reichlichen freien Flüssigkeit eine ausgedehnte Lazeration der Leber Grad IV, primär konservative Therapie, im Verlauf jedoch Laparotomie wegen einer Gallefistel

re das Potenzial zur Selbsttamponade im Kindesalter sehr groß. Speziell unter diesem Aspekt wurde das nichtoperative Management auch auf die Verletzungen von Nieren im Kindesalter übertragen. Der Erfolg dieser Behandlung wird durch vielfältige Studien belegt, zeigt aber noch eine verhältnismäßig große Variabilität bezüglich der Frequenz einer primären operativen Versorgung, die zwischen 2 und $33 \%$ beschrieben wird. Dabei liegt die primäre Nephrektomierate ebenfalls zwischen 2 und $28 \%$. Trotzdem muss darauf verwiesen werden, dass auch bei der operativen Versorgung einer Nierenverletzung im Kindesalter alle Operationstechniken zur Anwendung kommen sollten, die einen Teilorganerhalt ermöglichen. Unabhängig davon sind langfristige Verlaufskontrollen notwendig, da persistierende Perfusionsstörungen im Einzelfall auch eine Spätnephrektomie notwendig machen.

\section{Pankreasverletzungen}

Die Verletzung des Pankreas wird im Kindesalter selten beschrieben. Jedoch ist es durch die fixierte retroperitoneale Lage direkt vor der Wirbelsäule bei punktuell auftretender stumpfer Gewalt im Oberbauch für eine Verletzung im Kindesalter ebenso exponiert. Typischer Unfallmechanismus ist dabei die Lenkerverletzung durch Roller und Fahrrad. Somit ist bei klinisch sichtbarer Verletzungsmarke an der Bauchdecke prinzipiell an eine derartige Organverletzung zu den- ken (• Abb. 3). Klinisch imponiert sie oftmals mit zeitlicher Verzögerung, jedoch dann durch eine klassische Trias von Fieber, Leukozytose und erhöhter Amylase bzw. Lipase. Diese Laborwerte sind bei stationärer Aufnahme in bis zu $83 \%$ der Fälle bereits pathologisch erhöht. Neben der Sonographie hat hier die Kontrastmittel-CT zur diagnostischen Abklärung einen besonderen Stellenwert.

Besonders unter dem Aspekt der Therapieentscheidung ist auch im Kindesalter, wenn möglich, eine endoskopische retrograde Cholangiopankreatographie (ERCP) sinnvoll. Ist diagnostisch nachzuweisen, dass der Pankreasgang nicht verletzt ist, ist auch bei den Verletzungen der Bauchspeicheldrüse ein konservativer Therapieansatz Erfolg versprechend. Ist eine Läsion des Ductus pancreaticus nachzuweisen, steigt die Komplikationsrate bei konservativer Therapie auf nahezu $100 \%$ an [27]. In einer Studie von Cigdem et al. [2] aus dem Jahr 2011 wurden von 31 Pankreasverletzungen 25 konservativ behandelt. In dieser Gruppe traten bei 11 Patienten Komplikationen auf, die in 6 Fällen eine operative Intervention notwendig werden ließen. Somit muss insgesamt eingeschätzt werden, dass der konservative Therapieansatz bei Pankreasverletzungen, bei denen keine Mitverletzung des Ductus pancreaticus vorliegt, Erfolg versprechend sein kann. Jedoch muss insgesamt mit einer hohen Komplikationsrate und somit mit möglichen sekundären operativen Versorgungen gerechnet werden.
Verletzungen des Gastrointestinaltraktes

Den Verletzungen des Gastrointestinaltraktes im Rahmen eines stumpfen Bauchtraumas im Kindesalter kommt in der Hinsicht eine besondere Bedeutung zu, da diese möglicherweise primär häufig übersehen werden können. Insgesamt liegt die Frequenz derartiger Verletzungen lediglich bei 1,4-4,9\% aller Verletzungen. Es besteht eine klare Assoziation zu dem Zustand, ob Kinder als Pkw-Insassen korrekt oder nicht korrekt angeschnallt gewesen sind. Bei Vorliegen einer klinisch sichtbaren Gurtmarke im Bereich der Bauchdecke ist das Risiko für das Vorliegen einer Verletzung im Bereich des Gastrointestinaltraktes 4-mal höher als bei nicht korrektem Anschnallen [14]. Das klinische Beschwerdebild wird durch die sekundäre Peritonitis bestimmt. In den meisten Fällen kommt es zur Ausprägung eines Pneumoperitoneums, sodass im Rahmen der Diagnostik in diesen Fällen die konventionelle Röntgendiagnostik als Abdomenübersichtsaufnahme zum Nachweis der freien Luft uneingeschränkte Bedeutung hat. Zur Sicherung von retroperitonealen Verletzungen im Bereich des Duodenums ist auch hier die Kontrastmittel-CT indiziert. Therapeutisch lässt sich in diesen Fällen eine operative Versorgung der Verletzungen des Gastrointestinaltraktes nicht vermeiden. Bei Verletzungen des Duodenums sind die klassische Dekompression und Drainage indiziert. Bei Verletzungen des übrigen Intestinums finden die üblichen Operationstechniken wie Übernähung einer Perforation, Segmentresektion oder auch die passagere Anlage eines protektiven Enterostomas ihre Anwendung. Trotz der nicht selten verzögerten Diagnostik der Verletzungen des Gastrointestinaltraktes im Kindesalter ist die Gesamtmorbidität nicht durch einen späteren Operationszeitpunkt verschlechtert. 


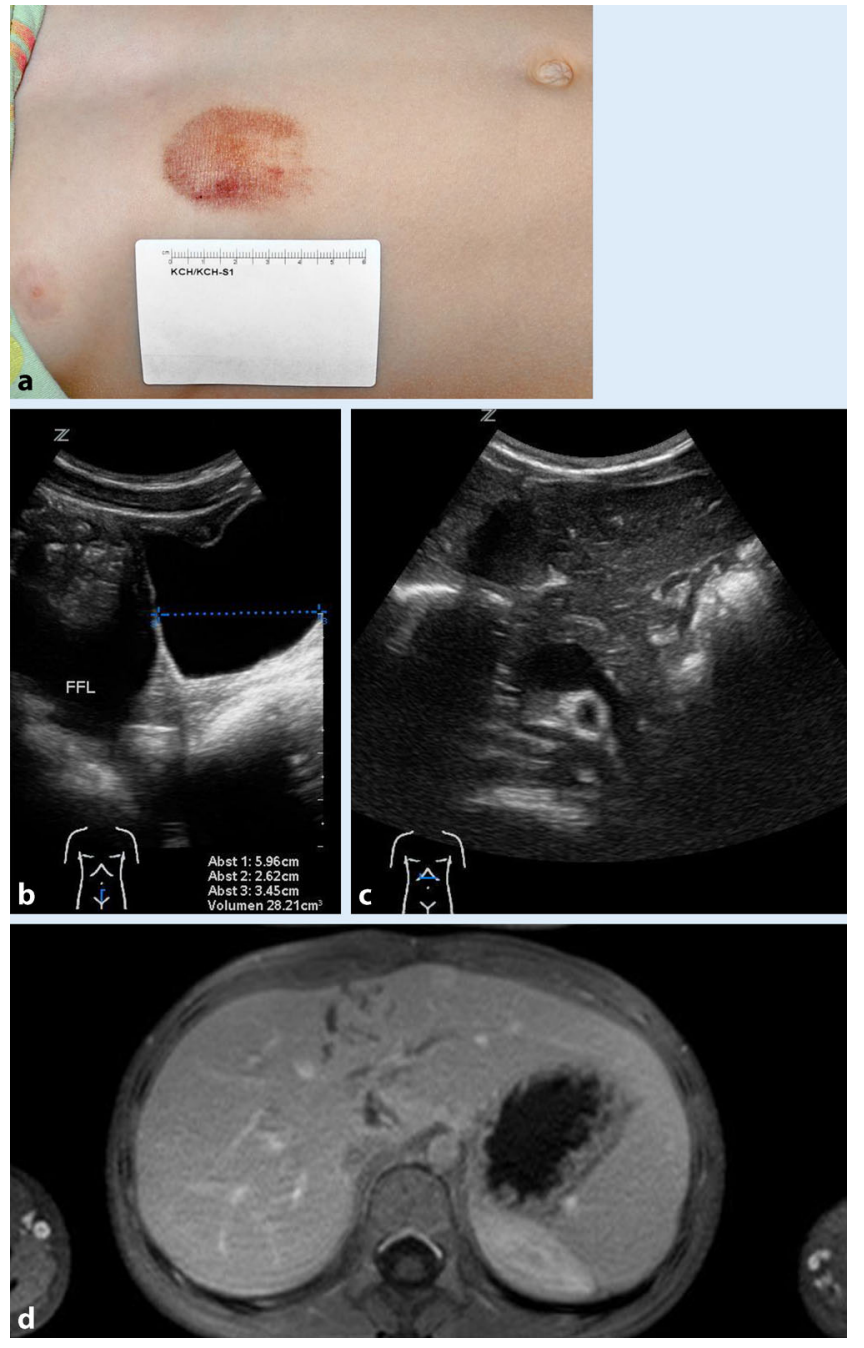

Abb. $3<$ Das 8-jährige Mädchen ist von den Fahrradpedalen abgerutscht und dabei mit dem Oberbauch auf den Fahrradlenker geprallt (aus [5]). a Klinisches Bild der Lenkerprellmarke im rechten Oberbauch. b Sonographie am Unfalltag mit reichlich freier Flüssigkeit retrovesikal.c Sonographie am Unfalltag mit ausgeprägter Leberläsion und Verdacht auf Pankreaskopfkontusion. d Magnetresonanztomographie (T2-Wichtung mit Kontrastmittel und Fettsättigung) zeigt die Leberruptur Grad IV

\section{Thoraxtrauma}

\section{Epidemiologie}

Das Thoraxtrauma im Kindesalter stellt hinsichtlich seiner Häufigkeit ein eher seltenes Ereignis dar. Charakteristisch für das Kindesalter ist das hohe $\mathrm{Maß}$ an Elastizität des Brustkorbes. Außerdem ist der knöcherne Thorax ein suffizienter Schutz, sodass Kinder meist nur nach einem schweren isolierten Thoraxtrauma oder wenn das Thoraxtrauma in Kombination mit anderen Verletzungen auftritt einer klinischen Behandlung zugeführt werden. So wurden beispielsweise im Children's Hospital in Pittsburgh - einem Level-I-Traumazentrum für Kinder - in 8 Jahren nur 105 Patienten im Alter bis zu 17 Jahren mit einem Thoraxtrauma behandelt. Dabei fällt auf, dass die Altersverteilung eine relative Homogenität
Dabei wurden insgesamt 3253 Unfälle erfasst, von denen bei 70 Patienten (1,3\%) Thoraxtraumata vorlagen [21].

Ungeachtet der relativen Seltenheit des Thoraxtraumas im Kindesalter handelt es sich aufgrund der Schwere der Verletzung per se oder aber durch die Kombination mit anderen Verletzungen nicht selten um lebensbedrohliche $\mathrm{Zu}$ stände. In der Pittsburgher Studie wird eine Letalität von 6,7\% (7 von $105 \mathrm{~Pa}$ tienten) beschrieben, die allerdings im Kleinkindalter mit 12,1\% (4 von $33 \mathrm{~Pa}$ tienten) überdurchschnittlich hoch ist. Die Prognose der Thoraxverletzungen wird dabei allgemein durch die Kombination mit anderen Verletzungen mit bestimmt [16]. Willital und Lehmann [25] beschreiben allein bei ca. $75 \%$ ihrer Patienten ein gemeinsames Auftreten mit einem stumpfen Bauchtrauma, und in der Pittsburgher Studie wurde in über der Hälfte der Patienten (53,3\%) eine kombinierte Form des Thoraxtraumas beschrieben, wobei insbesondere das gemeinsame Auftreten mit einem schweren Schädel-Hirn-Trauma mit einer schlechten Prognose assoziiert ist [16].

\section{Knöcherne Verletzungen}

Wird die Brustwirbelsäule von den Betrachtungen ausgeschlossen, dann ist der Thorax knöchern durch die Rippen und das Sternum begrenzt. Aufgrund der verminderten Mineralisation der Rippen im Kindesalter zeigen diese eine höhere Elastizität als beim Erwachsenen, sodass diese bei einem Thoraxtrauma ehergebogen als gebrochen werden. Trotzdem wird in der Literatur eine Frequenz von Rippenfrakturen bei Kindern mit einem Thoraxtrauma zwischen 25 und $68 \%$ angegeben [15, $16,20]$. Dabei treten diese in ca. einem Drittel isoliert auf. Die röntgenologische Diagnostik beiklinischem Verdacht einer Rippenfraktur hat eine niedrige Sensitivität, und ein normaler Röntgenbefund schließt das Vorliegen einer Rippenfraktur nicht sicher aus. So ist es zu erklären, dass nach stumpfem Thoraxtrauma eine erfolgte Rippenfraktur klinisch erst durch die Kallusbildung im Frakturbereich evident wird und dann unter Um- 


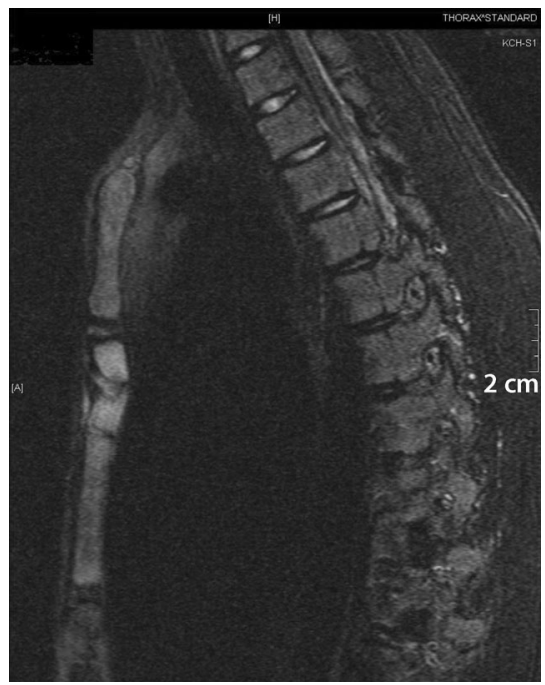

Abb. 4 \ Der 13-jährige Junge erlitt beim Turnen am Barren ein Flexionstrauma im Bereich des ventralen Thorax und zog sich dabei eine Sternumfraktur im mittleren Drittel zu. Die Darstellung mittels Magnetresonanztomographie zeigt die bereits in Abheilung befindliche Fraktur 2 Wochen nach Unfall. (Mit freundl. Genehmigung Schattauer Verlag, [4])

ständen sogar zu einem Tumorverdacht führen kann [1].

$\mathrm{Da}$ es sich in der überwiegenden Zahl der Fälle um stabile Frakturen handelt, besteht die Behandlung meist nur in einer Schmerztherapie, die die schmerzbedingt eingeschränkte Atemexkursion des Thorax aufheben soll und so eine normale Ventilation ermöglicht. Sehr selten führen Rippenserienfrakturen zu einem instabilen Thorax mit einer paradoxen Atmung, die dann durch eine Intubation und maschinelle Beatmung am besten zu stabilisieren ist. Nur im Ausnahmefall ist eine operative Intervention zur Stabilisierung der Rippen notwendig.

Wesentlich seltener als Rippenfrakturen - praktisch als Rarität - werden im Zusammenhang mit einem Thoraxtrauma im Kindesalter Frakturen des Sternums beschrieben. Da das Sternum in der Zentralachse des Körpers leicht zurückgesetzt liegt, ist es relativ geschützt vor traumatischen Einwirkungen. Daher wird als wesentlichste Ursache für eine Fraktur des Sternums eine direkte ungeschützte Gewalteinwirkung auf den Knochen angenommen, die, wenn sie insbesondere im Kleinkindalter auftritt, als Indiz für das Vorliegen einer Kindesmisshandlung gilt [26]. Dass dies nicht verallgemeinert werden kann, leitet sich aus der Beschreibung weiterer Unfallmechanismen im Zusammenhang mit dem Auftreten einer Sternumfraktur ab. So sind Sternumfrakturen ebenfalls nach starker muskulärer Anspannung, die zu einer Hyperextension des ventralen Thorax führen [9], oder aber nach einer plötzlichen forcierten Flexion der Brustwirbelsäule, wobei die Kräfte über die Claviculae auf das Sternum übertragen werden, beschrieben [6]. Alle diese Unfallmechanismen werden in einer Analyse von Hechter et al. [8] bestätigt, die in einer Periode von 10 Jahren 12 Patienten mit einer Sternumfraktur beschreiben. Diagnostisch ist die Fraktur durch ein tangentiales Röntgenbild des Sternums oder durch entsprechende Schnittbildverfahren wie CT oder Magnetresonanztomographie (MRT) darzustellen. Typischerweise befindet sich die Fraktur im mittleren Drittel des Sternums [8]. Es besteht meist keine wesentliche Dislokation der Fraktur, sodass ähnlich wie bei den Rippenfrakturen keine spezifische Therapie erfolgen muss.

In unserem eigenen Krankengut des letzten Jahres können wir über einen 13-jährigen Jungen berichten, der beim Turnen am Barren die Körperkontrolle verlor und im Schultergürtel nach ventral abknickte. Seit dieser Zeit klagte er über Schmerzen im mittleren Bereich des Sternums. Bei anhaltenden Beschwerden stellte der Junge sich nach 2 Wochen bei uns vor. Klinisch imponierten eine diskrete Vertiefung im Sternum sowie ein gewisser Druckschmerz. Unter dem Verdacht einer Thoraxwanddeformität wurde eine MRT des Thorax durchgeführt, die eine mäßig dislozierte Fraktur des Sternums im mittleren Drittel sicherte, wobei bereits Zeichen für ein Remodeling zu erkennen waren (๑Abb.4). Es erfolgten eine Entlastung mittels Rucksackverband für 2 weitere Wochen sowie eine Sportbefreiung für diese Zeit. Die Fraktur heilte problemlos aus.

\section{Lungenverletzung}

Wie bereits erwähnt, zeigt der kindliche knöcherne Thorax eine wesentlich höhere Elastizität als der des Erwachsenen, wodurch eine gewisse Protekti- on gegenüber knöchernen Traumafolgen gegeben ist. Andererseits wird allerdings durch die hohe Verformbarkeit des Brustkorbes bei einer Gewalteinwirkung diese Energie auf die intrathorakalen Organe weitergeleitet [22]. Folglich ist im Kindesalter nach einem Thoraxtrauma eine hohe Inzidenz an Lungenkontusionen zu beobachten, und diese stellt die häufigste Lungenverletzung im Kindesalter dar. In der Literatur wird sie mit einer Frequenz zwischen 37 und $61 \%$ angegeben $[16,22]$. Daher ist bei anamnestischen Anhaltspunkten für ein stumpfes Thoraxtrauma, aber ebenso bei einem stumpfen Oberbauchtrauma auch bei klinischer Unauffälligkeit eine Lungenkontusion abzuklären. Dies erfolgt in der Regel durch ein Thoraxröntgenbild a.p., stellt sich aber auch in einer Thorax-CT im Rahmen einer erweiterten Diagnostik bei Mehrfachverletzungen als unscharf begrenzte intrapulmonale Infiltrationen dar (• Abb. 5). Aufgrund der Minderbelüftung der Kontusionsherde innerhalb der Lunge besteht ein erhöhtes Infektionsrisiko. Daher wird eine prophylaktische Antibiotikatherapie zusammen mit einer Atemgymnastik als Standardtherapie durchgeführt.

Im Zusammenhang mit dem für die Lungenkontusion beschriebenen Unfallmechanismus muss auf das Auftreten von tracheobronchialen Verletzungen hingewiesen werden. Diese treten zwar prinzipiell sehr selten und praktisch immer im Rahmen eines schweren stumpfen Thoraxtraumas auf, aber in einer Metaanalyse von Kiser et al. [13] sind von 265 erfassten Fällen 222 auswertbar gewesen, von denen wiederum $50 \%$ im Alter unter 20 Jahren und $22 \%$ unter 10 Jahren vorkamen. Andererseits stellte sich in der Analyse von Autopsiebefunden bei Unfallopfern eine Inzidenz von tracheobronchialen Verletzungen nur zwischen 1 und 2,8\% dar, von denen aber ca. $80 \%$ die Klinik nicht mehr erreichten. Die meisten Verletzungen betreffen das Bronchialsystem in relativer Nähe zur Carina $[10,12,13]$.

Obwohl derartige Verletzungen in hohem Maß mit einer präklinischen Letalität einhergehen, die eher nicht durch die Verletzung per se, sondern durch die Schwere der Begleitverletzungen bedingt 


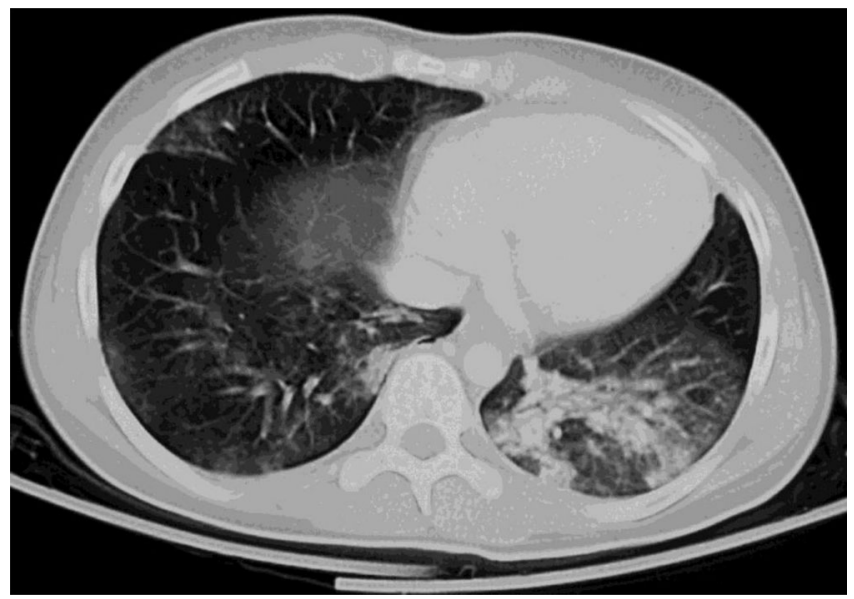

Abb. 5 \ Das 11-jährige Mädchen wurde als Fußgängerin von einer Straßenbahn erfasst und erlitt ein Polytrauma. Bei der primären Diagnostikmittels Spiralcomputertomographie stellten sich als Folge des stumpfen Thoraxtraumas multiple Lungenkontusionen beidseitig dar. (Mit freundl. Genehmigung Schattauer Verlag, [4])

ist, wird in der Klinik immer wieder eine diagnostische Latenz bis zu mehreren Tagen beobachtet. Klinisch fallen ein Hämatopneumothorax sowie ein mediastinales oder subkutanes Emphysem auf. Bei Verdacht muss der Befund bronchoskopisch gesichert und ggf. operativ versorgt werden. Hierbei besteht das Ziel, das Tracheobronchialsystem zu rekonstruieren, wobei im Ausnahmefall auch eine Segment- oder Lappenresektion in Betracht gezogen werden muss.

\section{Pneumothorax}

Im Gegensatz zu den tracheobronchialen Verletzungen stellt der Pneumothorax ein häufiges Ereignis nach einem Thoraxtrauma dar. In der Pittsburgher Studie wird die Häufigkeit mit 37,1 \% (39 von 105 Kindern) angegeben, von denen bei $23,1 \%$ (9 von 39 Kindern) ein Spannungspneumothorax und bei $25,6 \%$ (10 von 39 Kindern) der Pneumothorax ohne Begleitverletzungen isoliert aufgetreten ist. Ein kombinierter Hämatopneumothorax wurde bei $30,7 \%$ (12 von 39 Kindern) beobachtet. Dagegen wurde nur in 2 Fällen ein isolierter Hämatothorax beschrieben [16].

Das pathomorphologische Korrelat für den Pneumothorax stellt der Eintritt von Luft in den Pleuraspalt dar. Je nachdem, von wo die Luft hineingelangt, wird der Pneumothorax in einen inneren (Verletzung der Pleura visceralis
z.B. durch ein stumpfes Thoraxtrauma) und einen äußeren (Verletzung der Pleura parietalis durch penetrierende Verletzungen) Pneumothorax unterschieden. Hinsichtlich der Frage, ob eine Verbindung zur Umgebung besteht, unterscheidet man einen geschlossenen (keine Verbindung zur Außenluft) und einen offenen (dauerhafte Verbindung von Pleurahöhle zur Außenluft) Pneumothorax. Diese Unterscheidung ist in der Hinsicht von großer Bedeutung, da das Vorliegen eines offenen Pneumothorax eine lebensgefährliche Situation per se darstellt. Ursächlich dafür sind das sog. "Mediastinalflattern“ und die „Pendelluft“. Diese beiden pathophysiologischen Prozesse sind wesentlich für das Eintreten einer globalen Ateminsuffizienz mit kardialen Störungen bei einem offenen Pneumothorax und erklären somit die vitale Bedrohung des Patienten. Eine besonders bedrohliche Situation liegt beim Auftreten eines Spannungspneumothorax vor. Dabei tritt mit jeder Inspiration Luft in die Pleurahöhle der verletzten Thoraxseite ein, die bei Exspiration nicht wieder entweichen kann. Dadurch wird zunehmend das Mediastinum zur gesunden Thoraxseite verdrängt und komprimiert die gesunde Lunge, sodass auch hier eine globale Ateminsuffizienz mit gleichzeitiger kardialer Dekompensation droht und somit einen höchst lebensgefährlichen Zustand darstellt.
Aus dieser Darstellung leiten sich 2 am Unfallort notfallmäßig durchzuführende Maßnahmen ab:

- Jeder offene Pneumothorax ist in einen geschlossenen zu überführen und

- beim geringsten klinischen Verdacht auf das Vorliegen eines Pneumothorax (fehlende Thoraxexkursion, fehlendes Atemgeräusch, hypersonorer Klopfschall, Tachykardie, Einflussstauung) ist eine Thoraxdrainage anzulegen.

Bei einem instabilen Thorax müssen zusätzlich eine Intubation und Beatmung des Patienten erfolgen. Begleitend sollten immer eine Schockprophylaxe bzw. -therapie sowie eine Breitbandantibiotikabehandlung durchgeführt werden. Schließlich müssen in der Klinik die vielfältigen Ursachen für einen Pneumothorax diagnostisch abgeklärt und ggf. operativ versorgt werden.

\section{Penetrierende Verletzungen}

Penetrierende Thoraxverletzungen sind im Kindesalter äußerst selten. Von den 105 Kindern mit einem Thoraxtrauma der Pittsburgher Studie wurde lediglich bei 3 Kindern $(2,8 \%)$ eine derartige Verletzung behandelt [16]. Durch ein meist hohes Maß an Komplexität sind diese Verletzungen aber mit einer hohen Letalität assoziiert. Es handelt sich dabei überwiegend um Pfählungsverletzungen, aber auch Stich- oder Schusswunden werden beschrieben.

Die Erstversorgung derartig verletzter Kinder folgt den allgemein gültigen Prinzipien:

1. Die Fremdkörper werden in der Wunde belassen,

2. steriles Abdecken der Wunde, evtl. offenen Pneumothorax in einen geschlossenen überführen,

3. Intubation und Beatmung sowie

4. Transport in ein entsprechendes Traumazentrum.

Wenn es der Allgemeinzustand des Patienten zulässt, erfolgt hier die Diagnostik zur präoperativen Abschätzung der Komplexität der Verletzung. AnschlieBend ist eine explorative Thorakotomie 
mit Entfernung der Fremdkörper und Revision der Verletzungsbahn durchzuführen. Dabei wird bei Bedarf ein großzügiges Wunddébridement vorgenommen. Die rekonstruktive Versorgung intrathorakaler Organe sollte idealerweise durch ein interdisziplinäres Vorgehen gesichert werden. Bei Bedarf muss der Eingriff durch eine explorative Laparotomie ergänzt werden. Plastisch-chirurgische Rekonstruktionen von Weichteildefekten sollten immer einer sekundären Versorgung vorbehalten bleiben.

\section{Fazit für die Praxis}

- Bei Organverletzungen nach stumpfem Bauchtrauma im Kindesalter sind die häufigsten betroffenen Organe Niere und Milz, gefolgt von Leber, Pankreas und Gastrointestinaltrakt.

- In der Diagnostik sollte das Primat die sonographische Untersuchung haben. Die Kontrastmittel-CT sollte abhängig vom klinischen Befund und der therapeutischen Fragestellung durchgeführt werden.

- Das nichtoperative Management für isolierte Leber- oder Milzverletzungen der Stadien 1 bis 4 sowie Nierenverletzungen und Pankreasverletzungen ohne Läsion des Ductus pancreaticus stellt die primäre Behandlungsoption dar.

- Die Laparotomie sollte vermieden werden. Der Organerhalt hat das absolute Primat. Indikationen für eine primäre Operation stellen Kreislaufinstabilität, nachgewiesene Perforation eines Hohlorganes oder Peritonitis dar.

- Die überwiegende Mehrzahl der Thoraxverletzungen im Kindesalter hat leichteren Charakter und bedarf keiner operativen Intervention.

- Selten kommen schwere Verletzungen des Tracheobronchialsystems, penetrierende Thoraxverletzungen oder Hämatopneumothoraxe vor, die meist als Notfälle operativ zu versorgen sind.

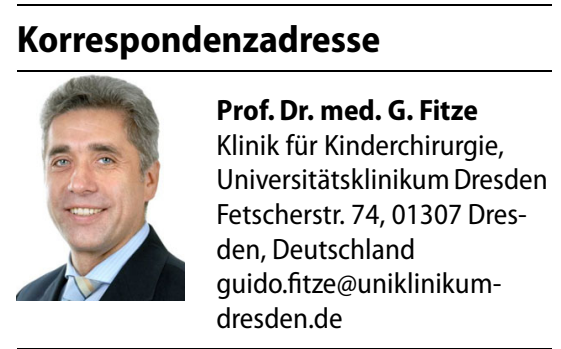

\section{Einhaltung ethischer Richtlinien}

Interessenkonflikt. G. Fitze gibt an, dass kein Interessenkonflikt besteht.

Dieser Beitrag beinhaltet keine vom Autor durchgeführten Studien an Menschen oder Tieren. Alle Patienten, die über Bildmaterial oder anderweitige Angaben innerhalb des Manuskripts zu identifizieren sind, haben hierzu ihre schriftliche Einwilligung gegeben. Im Falle von nicht mündigen Patienten liegt die Einwilligung eines Erziehungsberechtigten oder des gesetzlich bestellten Betreuers vor.

The supplement containing this article is not sponsored by industry.

\section{Literatur}

1. Bender TM, Oh KS, Medina JL, Girdany BR (1987) Pediatric chest trauma. JThorac Imaging 2:60-67

2. Cigdam MK, Senturk S, Onen A et al (2011) Nonoperative management of pancreatic injuries in pediatric patients. Surg Today 41(5):655-659

3. Davies DA, Pearl RH, Ein SH et al (2009) Management of blunt splenic injury in children: Evolution of the nonoperative approach. J Pediatr Surg 44:1005-1008

4. Fitze G (2007) Das Thoraxtrauma im Kindesalter. Kinder-Jugendmed 7:73-78

5. Fitze G (2016) Management der Organverletzungen bei Adoleszenten. Trauma Berufskrankh 18(Suppl. 2):111-S116. doi:10.1007/s10039-0150056-y

6. Fowler AW (1957) Flexion-compression injury of the sternum. JBone Joint Surg Br 39:487-497

7. Frumiento C, Vane DW (2000)Changing patterns of treatment for blunt abdominal splenic injuries: An 11-year experience in a rural state. J Pediatr Surg 35:985-989

8. Hechter S, Huyer D, Manson D (2002) Sternal fractures as a manifestation of abusive injury in children. Pediatr Radiol 32:902-906

9. Holderman $\mathrm{HH}$ (1928) Fracture and dislocation of sternum (report of 3 cases). Ann Surg 88:252-258

10. Kemmerer WT (1961) Patterns of thoracic injuries in fatal traffic accidents. J Trauma 1:595-599

11. Kiankhooy A, Sartorelli KH, Vane DW et al (2010) Angiographic embolization is safe and effective therapy for blunt abdominal solid organ injury in children. JTrauma 68(3):526-531

12. Kirsh MM, Orringer MB, Behrendt DM, Sloan H (1976) Management of trachoebronchial disruption secondary to nonpenetrating trauma. Ann Thorac Surg 22:93-101
13. Kiser AC, O'Brien MO, Detterbeck FC (2001) Blunt tracheobronchial injuries: Treatment and outcomes. Ann Thorac Surg 71:2059-2065

14. Lutz N, Arbogast KB, Cornejo RA (2003) Suboptimal restraint affects the pattern of abdominal injuries in children involved in motor vehicle crashes. JPediatr Surg 38:919-923

15. Meller JL, Little AG, Shermata DW (1984) Thoracic trauma in children. Pediatrics 74:813-819

16. Nakayama DK, Ramenofsky ML, Rowe MI (1989) Chest injuries in childhood. Ann Surg 210:770-775

17. Nellensteijn D, Porte RJ, van Zuuren W et al (2009) Paediatric blunt liver trauma in a Dutch level 1 trauma center. Eur J Pediatr Surg 19(6):358-361

18. Nellensteijn D, Greuter M, El Moumni $M$ et al (2016) The use of CT scan in haemodynamically stable children with blunt abdominal trauma: Look before yoe leap. Eur J Pediatr Surg 26(4):332-335

19. Ozturk H, Dokucu Al, Onen A et al (2004) Nonoperative management of isolated solid organ injuries due to blunt abdominal trauma in children A fifteen-year experience. Eur J Pediatr Surg 14(1):29-34

20. Rege VM, Deshmukh SS (1988) Major thoracic trauma in children. J Postgrad Med 34:93-99

21. Rollow A, Kehr A, Schneiders W, Roesner D, Zwipp $H$, Holch M, Reuter M (2006) Unfallumstände und Unfallfolgen. In: Schlag B, Roesner D, Zwipp H, Richter S (Hrsg) Kinderunfälle - Ursachen und Prävention. VS Verlag für Sozialwissenschaften, Wiesbaden, S68-93

22. Smyth BT (1979) Chest trauma in children. J Pediatr Surg 14:41-47

23. Stylianos S, APSA Trauma Committee (2000) Evidence-based guidelines for resource utilization in children with isolated spleen or liver injury. JPediatr Surg 35:164-169

24. Wessel LM, Scholz S, Jester I et al (2000) Management of kidney injuries in children with blunt abdominal trauma. J Pediatr Surg 35:1326-1330

25. Willital GH, Lehmann RR (2000) Thoraxtrauma. In: Willital GH, Lehmann RR (Hrsg) Chirurgie im Kindesalter, Bd. 1.Spitta, Balingen, S 1024-1033

26. Wojcik JB, Morgan AS (1988) Sternal fractures - the natural history. Ann Emerg Med 17:912-914

27. Wood JH, PatrickDA, BrunyJLetal (2010) Operative vs nonoperative management of blunt pancreatic trauma in children. J Pediatr Surg 45:401-406 